\title{
Q.
}

ISSN 2278 - 0211 (Online)

\section{A Review of Channels of Communication System on Project Delivery}

Olaniran, H. F.
Lecturer, Department of Building Technologgy, the Polytechnic, Ibadan, Nigeria

\section{Abstract:}

The importance of communication in the construction industry cannot be overemphasized. It is therefore, important that information is effectively communicated, understood and processed correctly. In this paper, we examined the various channels of communication to be carried out by construction professionals for a successful project delivery. The goal is to provide comprehensive information to site managers and all stakeholders in the building sector.

Keywords: Communication, channels, project, construction, professionals

\section{Introduction}

Communication is the transfer of information. Information is the generic term that embraces meaning such as knowledge, processed data, skills and technology (Cheng et al, 2001). Information transfer within construction environments presents special challenges. This is true of the construction industry, where interaction tends to be characterized by unfamiliar groups of people coming together for short periods before disbanding to work on other endeavours (Cheng et al., 2001). Communication is very vital for business and commercial activities. There is no doubt that any improvement in communication would lead to organization operating effectively. When there is good communication within an organization and between one organization and the other there will be improvement in motivation levels and construction process of the project. On the other hand, if communication is not carried out adequately it may result in demotivated workforce and lead to problems in construction. (Emmitt \& Gorse, 2003).

Construction communication, within an organizational context, is to convey an instruction to influence the actions and behaviors of others. It may also involve an exchange of, or request for information during a construction project (Dainty et al., 2006). It is therefore important and necessary for construction professionals to pass information or communicate at every stage of construction work, for a contract to be completed successfully. The workers and professionals involved should be well experienced, skilled, suitable and with the same mind set in order for the project goal to be attained. Communication among these professionals constitutes largely to a successful project execution. Construction is dynamic and of different elements effective communication becomes essential "for the successful delivery of performance goals (productivity, profitability and repeat working opportunities" (Dainty et al., 2006).

For construction process to be effective and efficient, it is a determinant of the quality of communication employed. There are four reasons mentioned in literature why communication needs to be improved. The first reason is that if the communication is improved between the project teams, building teams and between project manager and contractors, failure of the project will be reduced. Secondly, better communication at all levels could resolve to innovations and more good technical solutions. Thirdly, improvements in communication in the early stage of project would enhance the quality of the project as envisaged by all the people involved. Finally, communication during briefing might lead to making better decision, such as not taking hasty decision and better way of looking at the requirements needed for the project (Hoezen, 2011).

Considering that construction is such a fragmented, energetic and distinct sector, the challenges of communicating in an effective manner are greater than most other production environments. Relationships contract base on motivation, disagreement and conflict and a lack of mutual respect and trust, all combine to hinder open communication and render the role of the project manager extremely demanding and problematic. Nevertheless, addressing communication in the industry can be seen as a principal enabler for improving the industry in the future. Since the early 1940s, literature on communication in construction has appeared, mainly based on the situation in the UK (Emmitt and Gorse, 2003).

Construction is the physical execution of project, designed by the Architects or Engineers. The importance of construction industry to the public cannot be overemphasized, as buildings: offices are the commercial abode for most business owners, for children attending schools, people live in homes for safety, protection and convenience. All these buildings structures are being executed or erected by builders and other construction workers. It is therefore important and 
necessary for construction professionals to pass information or communicate at every stage of construction work, for a contract to be completed successfully. The workers and professionals involved should be well experienced, skilled, suitable and with the same mind set in order for the project goal to be attained. Communication among these professionals constitutes largely to a successful project execution. Construction is dynamic and of different elements effective communication becomes essential "for the successful delivery of performance goals (productivity, profitability and repeat working opportunities" (Dainty et al., 2006).

Many problems about communication have been reported, with a focus on inter communication within the construction sector, client-designer communication during the design stage and communication between and within a client and designers/ professionals in the course of construction procedures (Michael and Matous, 2015). In this paper, the necessary channels of communication are addressed in order for construction to be productive.

\section{Communication Channels}

Some of the most central and important factors militating against good performance of construction industry is its ineffective and inadequate channel of communication, barriers to harmonization between design and production process (Dainty et al., 2006). Effective communication between stakeholders in construction industry will contribute immensely to project delivery which is in contrast to poor communication which may affect decision making and consequently affect successful completion of the project. About sixty-five percent of contractor's rework is attributed to insufficient, inappropriate or conflicting information among stakeholders during the process of executing project plan (Newton, 1998). All these has been attributed to barriers in communication. Unfortunately, however, while there are reports of the causes of barriers to communication in the construction industries of industrialized and developed countries like the United States of America, Issa et al. (2003), Canada, Rivard (2000), Sweden, Denmark and Finland, Sampio et al. (2005) and New Zealand, Chassiako (2007), among others, comparatively little or no report exist for a developing country like Nigeria on causes of barriers of information. The findings are similar in respect of these industrialized and developed countries, the results, according to Austin (1995). These findings cannot be applicable to a third world country (like Nigeria) due to differences in the cultural, social, economic and regulatory environments of these countries (Jacklyn, 2011; Dawood et al., 2005).

In the last decade, many researchers' findings have showed poor project delivery within the construction industry in Nigeria in view of these reoccurring poor project delivery, researchers have focused their research on other factors causing or influencing poor performance such as purchase of construction material, quality assurance, health and safety of construction sites and availability of finance just to mention a few. Little or no attention is paid to communication channels as one of the major factors affecting poor construction project delivery.

In the process of project execution, transfer of information among project stakeholders come in varying forms, directions and mediums depending on communication flow which includes the sender and the receiver. Communication flow includes the upward flow of communication among management of organisations in a transaction. Also, there is lateral communication flow which occurs among customers and within project team members. Machinery needs to be put in place for further communication to take place, communication from superior to sub-ordinate- downward communication, communication among colleagues-horizontal flow or from sub-ordinates to superior-upward communication flow. After encoding a message, it has to be transferred through effective communication channels. This is the major challenge in construction due to a number of hindrances that comes across communication. An increasing use of project partnerships, strategic alliances and efforts to improve client satisfaction are increasing the demand for communication beyond organizational boundaries and to embrace project delivery structures and their supply chains. The reason for this is that the major participants within the construction industry do not operate in isolation they do liaise and interact with the suppliers and customers who surround them. For example, contracting organisations outsource larger percentage of their work and hire specialist design services when the need arises. These service providers are most at times external people to the organization but they are very important member of the project execution team. Effective communications channel is the bane for partnership between supply chain partners within and between the organisations involved.

Effective communication generates a hiccup for companies in their quest to execute a project especially for the managerial team who maintain a range of complex channels in different types of organisations. The efficacy of coordination is dependent on the medium through which information is transferred. In construction, a useful classification of how to communicate is either unidirectional flow or multidirectional flow. Unidirectional media facilitates one-way and linear flow of information from sender to receiver with no opportunities for feedback, the basics for unidirectional is the absence of feedbacks. This might include letters, drawings, emails or faxes between parties in the supply chain. In contrast, multidirectional media facilitate a reciprocal and two-way flow of information from sender to receiver with numerous opportunities for feedback. The examples for multidirectional communication include meetings, telephone calls or conferences. The aim of a project determines the medium of communication transfer, if the aim is to secure trust, mutuality and a spirit of cooperation among the project execution team then reciprocal media are preferable. Linear media has the ability to sabotage the environment fit for involvement and empowerment. However, the fact is that unidirectional medium of communication are cheaper and fastest ways to convey information and for this is reason unidirectional communication medium relied upon in projects execution, even where and when meetings might have been more appropriate. Without much ado, it is essential to note that the medium of communication used in construction is not always the most appropriate to ensure that information transmitted and understood. 
It is necessary that project managers have knowledge of innate human characteristics is essential to the interpersonal communication process. Cherry (1978) defines the barriers to effective communication as:

- Receivers have an expectation of messages from senders and assume the senders message is along with their thought even when it is different.

- Preconception of communicator exists by recipient

- Team work requires commitment; an aspect of commitment is that team members adhere to instruction by fellow team members than external instruction.

- Many words are homonyms, people interpret it differently from its initial meaning;

- Misinterpretation of non-verbal communication such as body language and other non-verbal signals'

- The process of listening and assimilating information is distorted by emotions, noise and other distraction.

- There is an inverse relationship between size of organization, therefore effective communication effective communication is less likely to occur in a large organization and vice versa.

Communicating using the reciprocal approach will enhance the likelihood for people to respond in the appropriate manner. The open communication is a synonym for interactive communication it allows for fair, equitable, honest and clear transfer of information and not manipulating it in favor of either of the sender or the receiver. Actually, in transfer of information the extent to which people need to have knowledge about a subject matter must be determined. In this regard, information is key to everyday activity, particularly when it deals with economic, financial and commercial issues. This is a proof that lack, inadequate information can lead to a number of challenges in project execution. Lima et al. (2005) identified that manipulation of information by project members in favour of their relation to unexpected problems is rampant. The effect is that problem resolution can be prolonged and made costlier. Thus, in these closed communications systems, the real agenda behind the transfer of information is kept secret and hidden, and this erodes the trust-based culture which is necessary for effective teamwork.

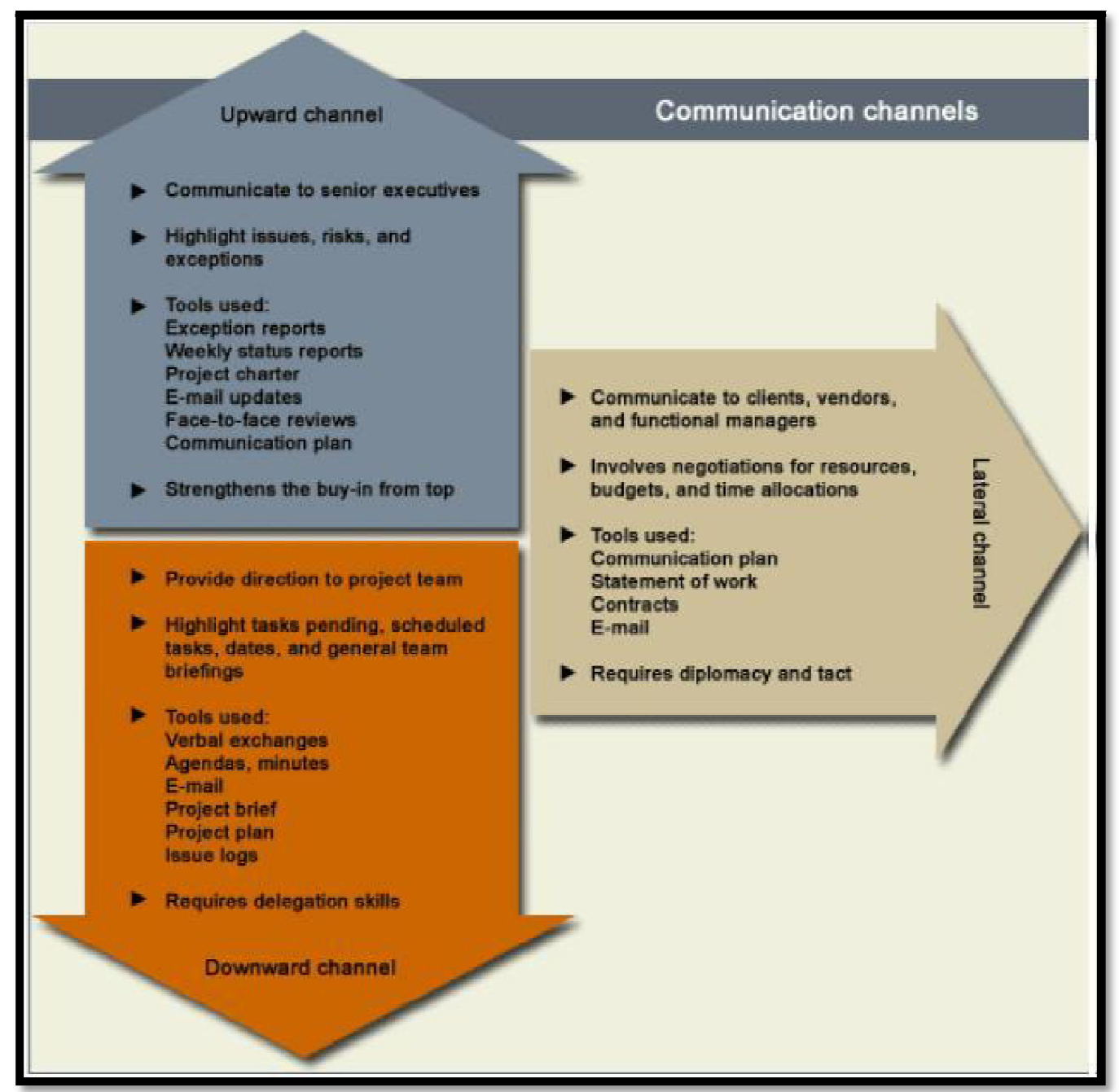

Figure 1: Communication Channels (Keyton, 2011) 


\section{Communication Models}

In communication, there is always a sender and a receiver, the number of receivers vary the receiver might be one person or more. Both parties have their own experiences, their perception, their ideas, just to mention a few, therefore may perceive and interpret information differently. The same event will always be interpreted in dissimilar ways by each communication participant. A simple communication model as Figure 2 shows how information is transmitted from sender to receiver.

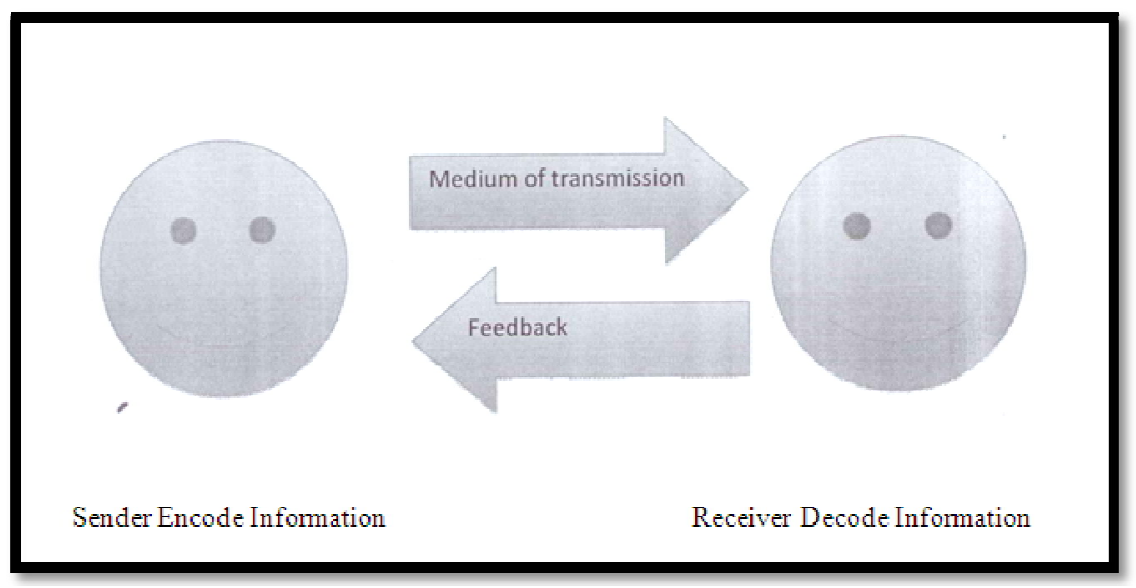

Figure 2: A Simple Communication Model (Mehra, 2009)

\subsection{Communication Pattern}

\subsubsection{The Sender}

Originates communication, usually has an information source and creates a message

\subsubsection{Encode}

Converting information into a message is encoding. The message should be properly encoded by the sender to make message easy to comprehend for the receiver. The knowledge, experience, perspective of the receiver should be taken into consideration in encoding and creating a message the person would be able to interpret in the way intended by the sender.

\subsubsection{Medium}

This is the means by which information is transferred between sender and receiver. It is also the communication method used to transmit the message. Message medium includes traditional mail, email, phone call, face-to-face or using gesture alone.

\subsubsection{Decode}

This is the interpretation and comprehension of message is encoded by sender. The sender uses his knowledge and understanding of the subject matter to decode this message, hence extra caution is required to interpret the message in by the receiver in the right context (sender's context).

\subsubsection{Receiver}

The one to whom information is sent.

\subsubsection{Feedback}

Receiver sends a feedback to sender to acknowledge that the information is received and understood. Sender may have to act further to ensure that the receiver understood the message by eliciting feedback that helps sender to assess whether receiver interpreted the message as intended.

\subsubsection{Status Reports}

This gives a summary of what the project stands at a specific point in time should follow the following organization:

\subsubsection{Accomplishments for Week or Month}

- Describe most important accomplishments. Relate them to project's Gantt chart.

- Describe other important accomplishments, one bullet for each. If any issues were resolved from the previous week or month, list them as accomplishments 


\subsubsection{Plans for Following Week or Month}

- Describe most important items to accomplish in the next month. Again, relate them to project's Gantt chart.

- Describe other important items to accomplish, one bullet for each.

\subsubsection{Issues}

A list of critical issues that showed up and are very essential

\subsubsection{Adjustment to Project}

This gives a detail of any changes to project. It includes the reason for adjustments, date of the adjustment and a brief description of the change.

\subsubsection{Minutes of the Meeting}

Is documentation of the last meeting, a plan of meeting to hold, it might sometimes include items of importance, crucial decisions, and action items.

\subsubsection{Request for Proposal (RFP)}

Document used to solicit proposals from prospective suppliers.

\subsubsection{Request for Quotation}

A document used to solicit quotes or bids from intending suppliers and contractor.

\subsubsection{Change Status Request}

Oral or written acts or omissions by someone with actual or apparent authority that can be construed to have the same effect as a written change order.

\subsubsection{Forecasts}

Used to predict future project status and progress based on past information and trends; especially project control and cost.

\section{Conclusion}

Various channels of communication exist in the construction industry for successful project delivery. Therefore, clearly defined and established structures must be on the agenda of team leaders and management before commencement of any project. The study therefore, concluded that there is a need for concerted strategies to stimulate the medium of communication in order to enhance an effective project delivery.

\section{References}

i. Austin, B. (1995). "Information management of construction projects", in "Integrated Construction Information", P. Brandon, M. Betts, (Editors), London, 291-315.

ii. Chassiakos, A. P. (2007). The Use of Information and Communication Technologies in Construction. Civil Engineering Computations: Tools and Techniques. Saxe-Coburg Publications, Stirlingshire, UK, 79-104.

iii. Cheng, E., H. Li, P. L, and Irani, Z. (2001). Network Communication in the Construction Industry. Within construction information is exceptionally diverse given the huge number of parties involved with the construction operations. Corporate Communication, 61-71

iv. Cherry, C. (1978) On human communication: A review, a survey, and a criticism. Cambridge and London: The MIT Press. 3rd ed.

v. Dainty, A., Moore, D. and Murray, M. (2006). Communication in Construction; Theory and Practice, Taylor and Francis.

vi. Dawood, N., Scott, D., Sriprasert, E., \& Mallasi, Z. (2005). The virtual construction site (VIRCON) tools: An industrial evaluation. Journal of information technology in construction (ITcon), 10(5), 43-54.

vii. Emmitt, S. and Gorse, C. (2003). Construction Communication, Blackwell Publishing Ltd.

viii. Hoezen, M.E.L. The Problem of Communication in Construction. University of Twente. Retrieved 23, December 2011.

ix. Issa, R. R. A., Flood, I., \& Caglasin, G. (2003). A survey of e-business implementation in the US construction industry. Journal of Information Technology in Construction (ITcon), 8(2), 15-28.

x. Jacklyn, W. (2011). Importance of construction communication. Microsoft Encarta Online Encyclopedia 2011. Retrieved June 20, 2011, from http:/ / www.stonemarkcm.com/ bartmendel.htm\#education

xi. Keyton, J. (2011). Communication and organizational culture: A key to understanding work experiences. Thousand Oaks, CA: Sage.

xii. Lima, C., El-Diraby, T., \& Stephens, J. (2005). Ontology-based optimisation of knowledge management in eConstruction. Journal of Information Technology in Construction (ITcon), 10(21), 305-327.

xiii. Mehra, S. (2009) Project communication Management. Accessed from the website: http:/ / www.scribd.com/ doc/ 7875707/ Project-Communication-Summary-by-Sachin-Mehra 
xiv. Michael, H.and Matous, P. (2015) Problem-solving discourse on an international construction site: Patterns and practicesEnglish for Specific Purposes, 18, 85-98.

xv. Newton, P. (1998). Diffusion of IT in the Building Construction Industry. CSIRO, Building for Growth Innovation Forum. Sydney.

xvi. Rivard, H. (2000). A survey on the impact of info tech in the Canadian Architecture, Engineeringand construction industry, journal of information technology in construction. (PP.37-56). http:// www.itcon.org/ 2000/ 3

xvii. Sampaio, A. Z., Henriques, P., \& Studer, P. (2005). Learning construction processes using virtual reality models. Journal of Information Technology in Construction (ITcon), 10(11), 141-151. 